\title{
Probabilistic reputation networks
}

\author{
Timothy Atkinson, ${ }^{1}$ Marius C. Silaghi ${ }^{1}$ \\ ${ }^{1}$ Florida Institute of Technology \\ atkinsot1999@my.fit.edu, msilaghi@ fit.edu
}

\begin{abstract}
A reputation system is proposed in the form of a Reputation Network based on the premise of availability of a model of the world as a Dynamic Bayesian Network where the network's posterior for one time slice is set as the prior probability in the next time slice. These networks are solutions with better overall properties than the original network reducing the complexity of using the results when the input data is random. Experiments are an extension of prior work detecting when P2P Group Owner (GO) negotiation unfairly makes one of the participants GO more often than the other. The decision process of who becomes GO can be modeled as a Bernoulli trial. Based on this model, we show that we can set the false positive rate, we can detect more nuanced cheating without extra nodes in the network to help us, and when the underlying distribution favors one element as GO, it will declare a positive and keep the positive set without needing a second interpretive layer. Furthermore, we demonstrate that the original properties of non-random data are maintained.
\end{abstract}

